\title{
An overview of possibilities for CAM machining
}

\author{
Andrei Marius Mihalache* \\ "Gheorghe Asachi" Technical University of Iasi, Department of Machine Manufacturing Technology, \\ Boulevard Dimitrie Mangeron 59A, Iasi, Romania
}

\begin{abstract}
There is an entire array of options when you are planning a CAM machining strategy. Knowing your equipment possibilities and adjusting your software options to them has become mandatory in terms of cost and time efficiency. This paper aims to present the main options for most common software solutions used today in modern mechanical engineering which can be applied to the existing hardware manufacturing equipment found in enterprises or educational centers.
\end{abstract}

\section{Introduction}

Today's CAM software solution have to provide tools in such a way that programmers have at their disposal all the instruments needed to get the job done. But CAM solutions have to encapsulate the same features for a variety of modern NC centers and it has to address each one in the same manner. Whether it is Mazak or Hurco NC centers, or Fanuc versus Sinumerik controllers CAM solutions must know their capabilities, acknowledge them and take advantage of their capabilities [1]. A modern CAM software solution knows about high speed machining versus prolonged tool life and is able to provide support to the programmer in sense of manufacturing strategies. Knowledge about software capabilities and the strategies that may be applied as well as the fact that it can be implemented onto existing machining equipment is a must for a senior programmer [2-3]. This paper aims to provide useful information about milling CAM operations and their machining optimization strategies and how software aids at their implementation.

\section{General capabilities}

All modern CAM software solution have certain capabilities that form industry standards. They are about the possibility to provide fast and accurate results under user friendly settings, about encapsulation of different operations in batches and export them in such a manner that they would be available offline, the possibility for interchangeable settings and standard friendly formats as well as the possibility to program in a certain manner that would be recognizable by other software solutions in terms of parameter setup and the changes that are made upon them [4].

CAM software solutions have to understand the machining strategy that the programmer had in mind and to be able to output the right numerical code for the right equipment [5].

\footnotetext{
*Corresponding author: andrei.mihalache@yahoo.com
} 


\subsection{Fast results}

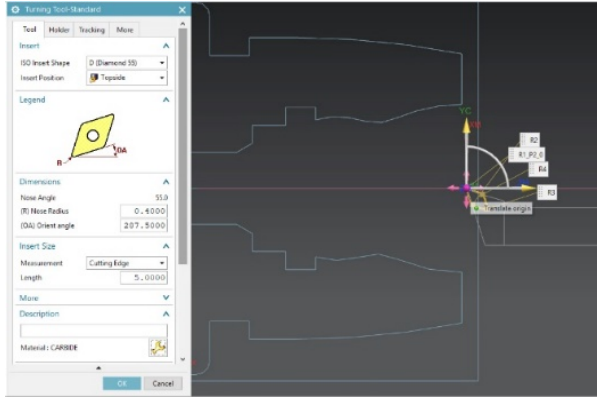

Fig. 1. Intuitive setup for faster results.
This means that the system allows fast programming providing all the related tools in sequenced windows for a specific task with minimal errors. The software solution has automated functions dependent of each other that self-adjust on modification of various parameters. It provides a task oriented management system and all the way indications and descriptions of issues or errors in such a way that input data will be clear and within range.

There is a progress status displayed and any changes are automatically propagated throughout the system. There is also the possibility to copy the entire setup or just machining steps or tools or features and to easy adjust them to the required need. The system automatically recognizes erroneous entries and highlights them.

Modern CAM software solutions allow multiple sequences of different projects thus making it possible for a project to be let's say processed as other is being programmed [6]. On the other hand a management window allows multiple machining strategies to be part of the same job listed according to the programmer needs. It incorporates the CAD model with all relevant links available.

\subsection{Compound job}

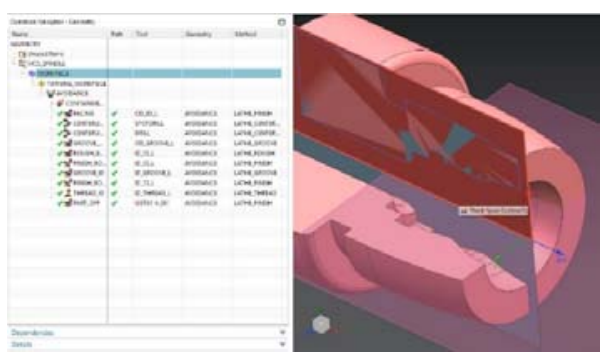

Fig. 2. Batch of multiple jobs.
A machining sequence encapsulate all the machining processes as a single task or job. Jobs may be then introduced in an array thus the name of compound job list giving users the opportunity to structure them considering position, tool orientation or the machining step itself. The jobs are available inside a network making them visible or hidden to specific users according to the needs.

They can be grouped and exported as a batch and may contain thousands of individual tasks that are available offline as a package.

\subsection{Associative programming}

The software allows users to work on copies of the same job because often it is the case that just few parameters have to be adjusted for a new machining as the strategy stays the same. All the parameters are linked inside a reference job and any modification made to the original will be inherit by the parameters untouched in the copies that have been made. But copy related parameters may be unlinked and modified without affecting any other as well.

Furthermore setups from a particular software solution may be exported with all relevant information including the $\mathrm{CAD}$ model that has been used in a general format that is readable by other CAM related solutions as well as for offline use [7]. 


\subsection{Parameter programming}

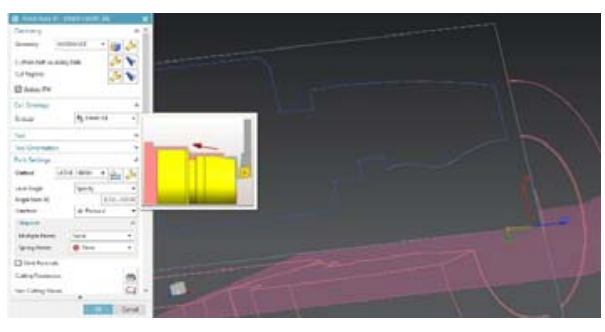

Fig. 3. Parameter extended setup.

It also allows definition of multiple zero points inside a compound job.

\section{CAM milling capabilities}

\subsection{Face milling}

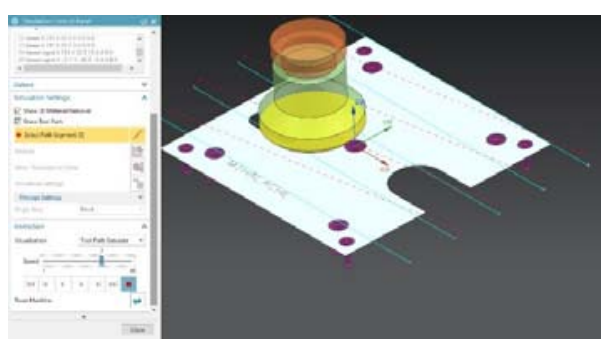

Fig. 4. Face milling setup.

\subsection{Pocket milling}

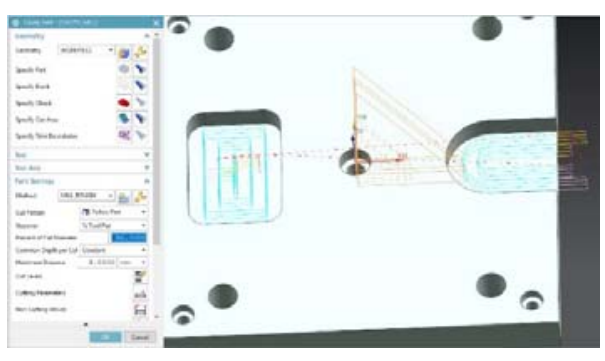

Fig. 5. Pocket milling setup.
Programming in sequence allows the user to adjust each parameter for a flexible workflow. The user is aware at all-time about dependencies and how changes affect the overall process as variables are modified. This strategy also allows the software to implement changes about different setups or equipment as defining new zero points, accommodate additional clamping or adjusting tool positions [8]. 


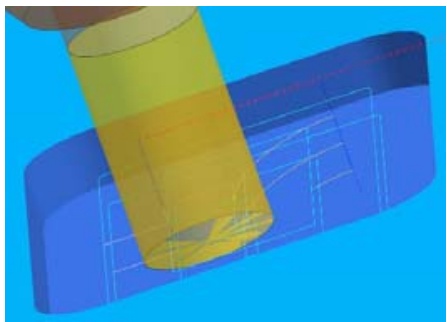

Fig. 6. Automatic feature recognition.

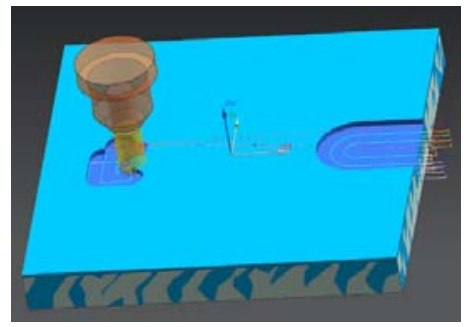

Fig. 7. Complete machining of the bottom.

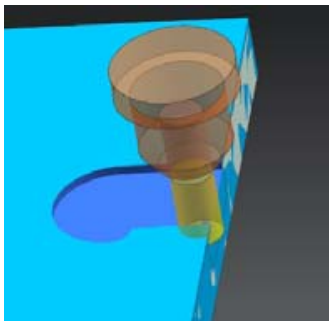

Fig. 8. Supports 2D controller cycles.

\subsection{Contour milling}

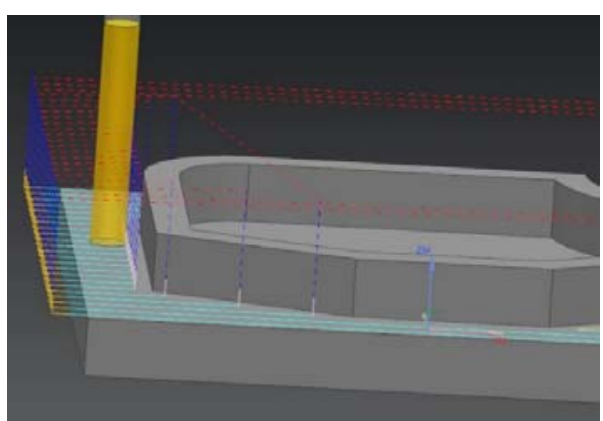

Fig. 9. Contour milling tool path.
This operation is used when milling complex contours. The software takes into account G41/G42 tool paths compensation as the tool can start machining from the center path or follow the contour's borderlines. It also detects areas that cannot be machined inside the same operation, bottle-necks, wrong retract moves or self-cuts within the containment zone. There are also aids in form of optimizations regarding the sorting of tool paths which are very helpful when dealing with multiple contours.

The software provides tips and identifies possible starting points taking into account overall tool moves and how to minimize them [10].

Also, the tool moves may be optimized in terms of Approach and Retract in such ways that we would have minimum distances or collision-free machining to ensure that infeed and transition movements are performed in the most suitable areas of the analyzed contours.

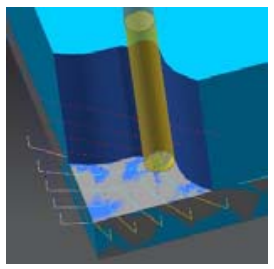

Fig. 10. Self-cut and bottleneck checkup.

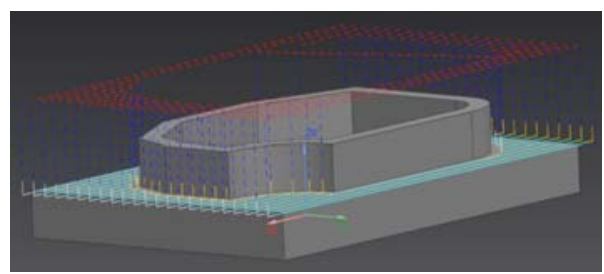

Fig. 14. 2D multiple lateral infeed.

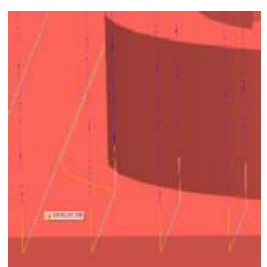

Fig. 12. Fillet outside edges.

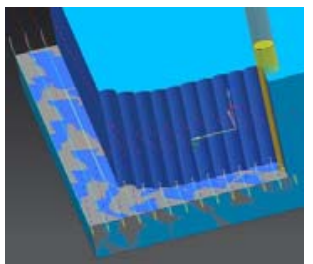

Fig. 13. Z-infeed trimming against the model.

Out of optimization functions the user has the possibility to choose from Self-cut and bottleneck check-up (see Figure 10), Spiral machining down the bottom (see Figure 11), Fillet outside edges (see Figure 12), Z-infeed trimming against the model (see Figure 13) and 2D multiple lateral infeed (see Figure 14). 


\subsection{Rest machining}

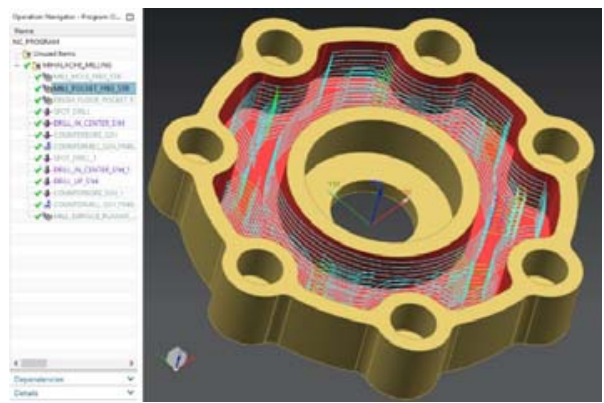

It is not always possible to machine contours or pockets using the same tool as you did for large surfaces. This involves a more accurate approach thus a new strategy. It computes distinct tool paths for smaller tools. The software points out and highlights the areas that will be left unprocessed and proposes alternatives. Areas may not be only inside closed contours but the software finds them even if they are on different geometries.

Fig. 15. Rest machining setup.

This strategy ensures a perfect finish of all areas by combining tool changes where they are necessary keeping in mind at all time a cost-time ratio [3]. As machining strategies we could mention Residual stock machined with a previous cycle or Tangential infeed for best contours.

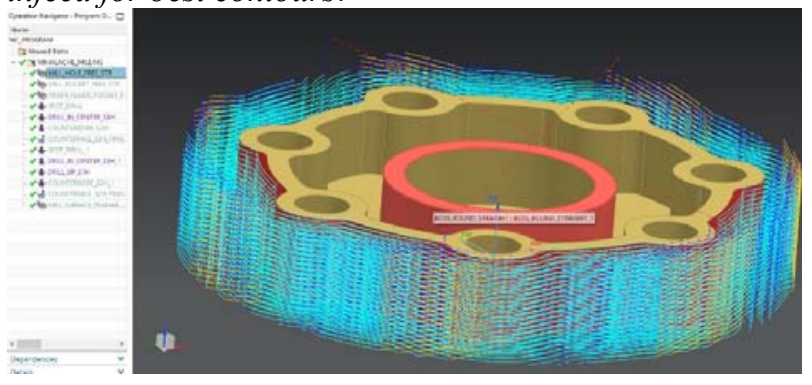

Fig. 16. Residual stock machined with a previous cycle.

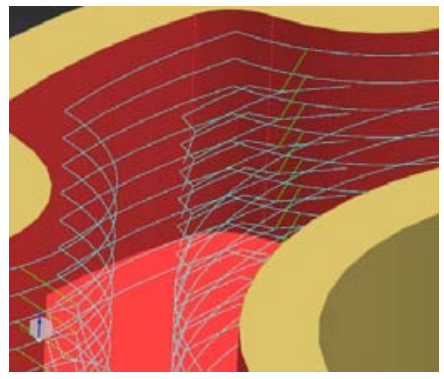

Fig. 17. Tangential infeed for best contours.

\subsection{Drilling}

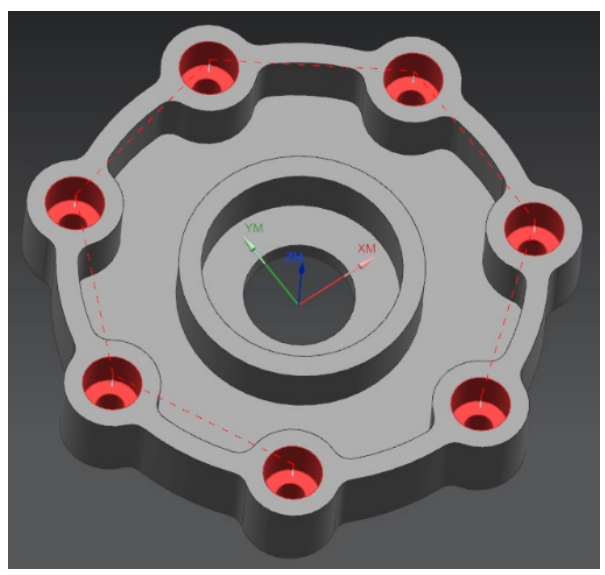

Fig. 18. Identified drilling geometric features.
The software offer possibilities for Centering, Simple drilling, Deep hole drilling, Deep drilling, Drilling with chip break, Reaming and Boring, Thread milling. Those are especially helpful when they are combined with so-called macro-milling. The machining can occur on its own or combined with other operations in a single job, compound jobs, subroutines or cycles [5]. They are available also for automatic recognition programming. From the optimization point of view the user may consider for Drilling the shortest path, Xparallel (see Figure 19) or Helical drilling with freely definable pitch (see Figure 20). 


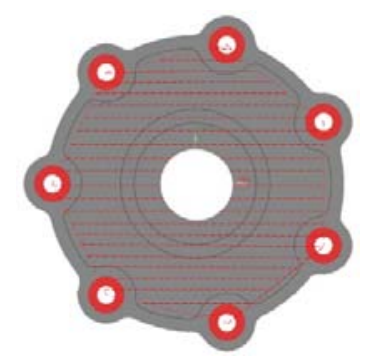

Fig. 19. Drilling on the shortest path.

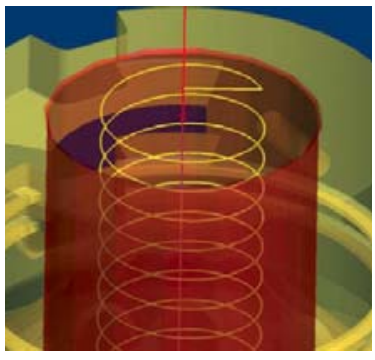

Fig. 20. Helical drilling.
The machining optimization strategy allows the user to define the pitch of the spiral. The other strategies are suitable as their names imply for deep holes or threads both exterior and interior.

\section{Conclusions}

In case of machining using CAM related optimization strategies there are various possibilities that carry different names on different software solutions but essentially do the same thing. For each operation the programmer is provided with several options that allows him to implement the desired policy whether is about prolonging tool life by using smaller feeds or is about cost-time efficiency by using more of the tool's length deeper into the blank.

When dealing with the same geometrical feature the CAM software solution allows the user to adjust the avoidance planes in such way that the tool does not have to withdraw to a safety position after each operation. Even more if the user has to deal with multiple machining operation patterns which would imply the use of several tool paths and inclinations, the software provides additional retraction positions in order to further reduce path lengths. The collision free politics is also present in case of changes between machined features and the machining planes. When detected, an automatic collision-free plane is created in order for the tool to be able to withdraw without any collision occurrence.

Furthermore being able to program with available tools and make the best of it is something that defines a good programmer. There are many tweaks that can be applied in any machining strategy but in the end it all comes to knowledge, skills and available logistics.

\section{References}

1. V. Merticaru et al., Convergent use of advanced CAD/CAE/CAM capabilities for sustainable integrated engineering 12, 43-48 (2014)

2. A.S.M. Hoque, Computers \& Industrial Engineering 66, 988-1003 (2013)

3. G. Musca, A. Mihalache, L. Tabacaru, IOP Conference Series-Materials Science and Engineering 161 (2016)

4. T. Dodoka, et al., Procedia Engineering 192, 113-118 (2017)

5. G. Musca, E. Musca, V. Merticaru, DAAAM Proceedings 8, 1485-1488 (2009)

6. M.I. Ripanu, et al., Applied Mechanics and Materials 371, 153-157 (2013)

7. P. Theodoroua, Omega 36, 107-121 (2008)

8. D. Luca, V. Schiopu, Journal of Optoelectronics and Advanced Materials 17, 997-1003 (2015)

9. G.M. Minquiz, Procedia CIRP 14, 581-586 (2014)

10. G. Musca, L. Tabacaru, Performance in design with SolidEdge ST and technical data management in product design (translation from Romanian), (PIM, 2010) 